This is the final peer-reviewed accepted manuscript of:

Zattoni, E., Perdon, A. M. and Conte, G. (2016) ‘Disturbance Decoupling With ClosedLoop Modes Stability in Switched Linear Systems', IEEE Transactions on Automatic Control, 61(10), pp. 3115-3121.

The final published version is available online at:

https://doi.org/10.1109/TAC.2015.2498123

Rights / License:

The terms and conditions for the reuse of this version of the manuscript are specified in the publishing policy. For all terms of use and more information see the publisher's website.

This item was downloaded from IRIS Università di Bologna (https://cris.unibo.it/)

When citing, please refer to the published version. 


\section{Disturbance Decoupling with Closed-loop Modes Stability in Switched Linear Systems}

\author{
Elena Zattoni*, Anna Maria Perdon`, and Giuseppe Conte ${ }^{\diamond}$
}

\begin{abstract}
This work deals with the problem of rendering the output of a switched linear system insensitive to a disturbance input, with the requirement that the dynamics of the closed-loop system be exponentially stable for all switching signals with a sufficiently large dwell time. A necessary and sufficient condition for the problem to have a solution is shown. A complete computational procedure to check the solvability condition and to synthesize the compensation scheme is provided. A worked-out example is presented with the aim of showing the effectiveness of the devised method.
\end{abstract}

Index Terms-Disturbance decoupling, switched linear systems, dwell time, exponential stability.

\section{INTRODUCTION}

In the last decades, switched systems have proved to be a powerful tool to handle control problems stated for systems featuring different modes of operation and a signal designating the active mode at each instant of time. $\mathcal{H}_{2}$ and $\mathcal{H}_{\infty}$ control, LQR optimal control, and output regulation are classic synthesis problems recently solved for switched systems [1]-[4]. In addition to these problems, disturbance decoupling, which, in its various formulations - depending on the information available on the to-be-decoupled signals [5], [6] — has been the object of a wide literature, has lately been considered for switched linear systems as well [7]-[10].

To be more specific, all the works just mentioned solve the problem of structural decoupling: i.e., they provide conditions on which the system output is zero for any admissible disturbance, on the assumption that the system initial state is the origin of the state space. As to stability, the issue lends itself to various approaches. Thus, a different solution can be found in each of the papers mentioned above except [9], which is exclusively focused on structural decoupling and introduces both a mode-dependent and a mode-independent static state feedback. In particular, [7] and [8] take into account the requirement that quadratic stability of the resulting closed-loop system be obtained with a suitable choice of the switching rule: in [7] a sufficient condition is shown, while in [8] an obstruction is pointed out, so that a necessary condition for the problem to be solvable with stability is provided. In [10], constructive, sufficient conditions for quadratic stability under arbitrary switching of the closed-loop dynamics are proved.

However, quadratic stability under arbitrary switching is only a sufficient condition for asymptotic stability and it can be rather conservative [11]. Hence, in order to reduce the level of conservatism intrinsic in the search for common quadratic Lyapunov functions, scientific research has developed along various directions, still in the framework of Lyapunov theory [12]. On the other hand, it has been shown that switched systems may not be asymptotically stable under arbitrary switching, but may enjoy this property for some classes of switching signals, such as those satisfying specific time-domain restrictions [13]-[16]. These considerations motivate the investigation of the disturbance decoupling problem carried out in this work, where exponential stability of the closed-loop dynamics is achieved for all switching signals with a sufficiently large dwell-time [17], by ensuring that all the closed-loop modes are made asymptotically stable by the same state feedbacks that render the output insensitive to the disturbance.

Manuscript version: November 3, 2015

* Department of Electrical, Electronic, and Information Engineering "Guglielmo Marconi", Alma Mater Studiorum - University of Bologna, 40136 Bologna, Italy (e-mail: elena.zattoni@unibo.it)

${ }^{\diamond}$ Department of Information Engineering, Polytechnic University of Marche, 60131 Ancona, Italy (e-mail: perdon@univpm.it, gconte@univpm.it)
The methodology developed in this work ensues from the geometric approach [18], [19]. Indeed, the use of geometric tools has been the mainstream in extending the solution of the disturbance decoupling problem from linear time-invariant systems [18], [19] to different classes of dynamical systems, like nonlinear systems, implicit systems, systems over rings, time-delay systems, and LPV systems [20]-[24]. In this context, the contribution of this paper is to provide a necessary and sufficient condition that, in the case of switched linear systems, completely and concisely characterizes solvability of the disturbance decoupling problem, with exponential stability of the closed-loop dynamics for all switching signals whose dwell time is large enough. Such condition involves a new geometric subspace, whose definition stems from elaborating further the geometric notions of robustness and internal stabilizability of a controlled invariant subspace. This subspace — which, to be precise, is the maximal robust controlled invariant subspace with the property of being internally exponentially stabilizabile for all switching signals with a sufficiently large dwell time - proves to fit with the hybrid nature of a switched linear system perfectly. As will be shown in the work, robustness of the controlled invariant subspace is a structural property that masters the multiplicity of the modes, while internal stabilizability handles the occurrence of switches and its impact on stability. The introduction of this new geometric object and the statement of a necessary and sufficient condition for the problem solution, relying on the structural and stability properties of this subspace, substantially distinguishes the contribution of this work from that of [25], where the condition provided was only sufficient, being based on a subspace which only featured structural properties.

The paper is organized as follows. In Section II, the disturbance decoupling problem with exponential stability under sufficiently slow switching is stated. The geometric notions functional to the problem solution are presented in Section III. The necessary and sufficient condition for the existence of a solution is shown in Section IV. A numerical example is worked out in Section V. Section VI contains the conclusions.

Notation: $\mathbb{R}, \mathbb{R}^{+}$, and $\mathbb{Z}^{+}$stand for the sets of real numbers, nonnegative real numbers, and nonnegative integer numbers, respectively. Matrices and linear maps are denoted by slanted capital letters, like $A$. The image and the kernel of $A$ are denoted by $\operatorname{im} A$ and $\operatorname{ker} A$, respectively. The transpose of $A$ is denoted by $A^{\top}$. Sets, vector spaces, and subspaces are denoted by calligraphic letters, like $\mathcal{X}$. The restriction of a linear map $A$ to an $A$-invariant subspace $\mathcal{J}$ is denoted by $\left.A\right|_{\mathcal{J}}$. The map induced by $A$ on the quotient space $\mathcal{X} / \mathcal{J}$ is denoted by $\left.A\right|_{\mathcal{X} / \mathcal{J}}$. The symbols $I$ and $O$ respectively denote an identity matrix and a zero matrix of appropriate dimensions. The symbol $\uplus$ denotes the union with repetition count.

\section{PRoblem StATEMENT}

Consider the continuous-time switched linear system

$$
\Sigma_{\sigma(t)} \equiv\left\{\begin{array}{l}
\dot{x}(t)=A_{\sigma(t)} x(t)+B_{\sigma(t)} u(t)+H_{\sigma(t)} h(t), \\
e(t)=E_{\sigma(t)} x(t),
\end{array}\right.
$$

where $t \in \mathbb{R}^{+}$is the time variable, $x \in \mathcal{X}=\mathbb{R}^{n}$ is the state, $u \in \mathcal{U}=\mathbb{R}^{p}$ is the control input, $h \in \mathbb{R}^{m}$ is the disturbance input, and $e \in \mathbb{R}^{q}$ is the to-be-controlled output, with $p, m, q \leq n$.

The modes of $\Sigma_{\sigma(t)}$ are the linear time-invariant systems

$$
\Sigma_{i} \equiv\left\{\begin{array}{l}
\dot{x}(t)=A_{i} x(t)+B_{i} u(t)+H_{i} h(t), \quad i \in \mathcal{I}, \\
e(t)=E_{i} x(t),
\end{array}\right.
$$

of the finite set $\left\{\Sigma_{i}, i \in \mathcal{I}\right\}$, where $\mathcal{I}=\{1,2, \ldots, N\}$. The matrices $A_{i}, B_{i}, H_{i}$, and $E_{i}$, with $i \in \mathcal{I}$, are constant real matrices of suitable dimensions. Moreover, $B_{i}, H_{i}$, and $E_{i}$ are assumed to be full-rank for all $i \in \mathcal{I}$. The sets of the admissible controls and disturbances are 
respectively defined as the sets of all piecewise continuous functions $u(t)$ and $h(t), t \in \mathbb{R}^{+}$, with finite values in $\mathbb{R}^{p}$ and $\mathbb{R}^{m}$.

The switching signal is a piecewise constant function $\sigma: \mathbb{R}^{+} \rightarrow \mathcal{I}$, $t \rightarrow i$, so that the active mode at any time $t \in \mathbb{R}^{+}$is $\Sigma_{i}$, with $i=\sigma(t)$. The discontinuity points of the switching signal - i.e., the switching times - are assumed to be in finite number in any finite time interval. The sequence of the switching times is denoted by $\left\{t_{\ell}, \ell \in \mathbb{Z}^{+}\right\}$and $\tau=\inf _{\ell \in \mathbb{Z}^{+}}\left\{t_{\ell+1}-t_{\ell}\right\}$ is a positive real number called dwell time ( $\tau=\infty$ is assumed for the special case of constant switching signals). Moreover, the set of all switching signals with $\tau \geq \tau_{d}$, where $\tau_{d}$ is a finite positive real constant, is denoted by $\mathscr{S}_{\tau_{d}}$. The switching signal is assumed to be accessible for measurement - hence, available to the compensation scheme.

Let $F_{\sigma(t)}: \mathcal{X} \rightarrow \mathcal{U}$ denote the switched state feedback defined by the indexed set $\left\{F_{i}: \mathcal{X} \rightarrow \mathcal{U}, i \in \mathcal{I}\right\}$ and the switching signal $\sigma(t)$. Hence, the closed-loop system is described by the continuous-time switched linear system

$$
\Sigma_{F, \sigma(t)} \equiv\left\{\begin{aligned}
\dot{x}(t) & =\left(A_{\sigma(t)}+B_{\sigma(t)} F_{\sigma(t)}\right) x(t)+H_{\sigma(t)} h(t), \\
e(t) & =E_{\sigma(t)} x(t),
\end{aligned}\right.
$$

with the modes

$$
\Sigma_{F, i} \equiv\left\{\begin{array}{l}
\dot{x}(t)=\left(A_{i}+B_{i} F_{i}\right) x(t)+H_{i} h(t), \quad i \in \mathcal{I} . \\
e(t)=E_{i} x(t),
\end{array}\right.
$$

The continuous-time switched linear system $\Sigma_{\sigma(t)}$ is said to be exponentially stable (exponentially stabilizable by state feedback, respectively) over $\mathscr{S}_{\tau_{d}}$ if it is exponentially stable (if there exists a switched state feedback $F_{\sigma(t)}$ such that the closed-loop system $\Sigma_{F, \sigma(t)}$ is exponentially stable, respectively) for all switching signals in $\mathscr{S}_{\tau_{d}}$. This property will be briefly referred to as exponential stability (exponential stabilizability, respectively) under dwell-time switching. Hence, the problem of disturbance decoupling with exponential stability of the closed-loop system under dwell-time switching is stated as follows.

Problem 1: Given the switched linear system $\Sigma_{\sigma(t)}$, find a switched state feedback $F_{\sigma(t)}$, such that

$\mathcal{R} 1$. the closed-loop switched system $\Sigma_{F, \sigma(t)}$, with initial state $x(0)=0$, has identically zero output, for all admissible disturbance inputs, and

$\mathcal{R} 2$. the closed-loop modes $\Sigma_{F, i}$, with $i \in \mathcal{I}$, are asymptotically stable.

In Problem 1, Requirements $\mathcal{R} 1$ and $\mathcal{R} 2$ express the structural and the stability specifications, respectively. As to Requirement $\mathcal{R} 1$, it is worth noting that perfect decoupling can still be achieved when the initial state is not zero, but belongs to a certain subspace of the state space. Such subspace will be exactly determined and pointed out in Remark 7. As to Requirement $\mathcal{R} 2$, it is worth noting that closed-loop asymptotic stability of all modes implies that there exists a positive constant $\tau_{d}$ such that the closed-loop switched system $\Sigma_{F, \sigma(t)}$ is exponentially stable over $\mathscr{S}_{\tau_{d}}$, by virtue of [17, Lemma 2]. Moreover, as to Requirement $\mathcal{R} 2$, that - as observed above expresses exponential stability under dwell-time switching, it is worth mentioning that when the switching signals are time-driven (i.e., they are independent of the state trajectory), which is the case considered in this work, exponential stability of the switched linear system is equivalent to uniform asymptotic stability [26].

\section{USEFUl NOTIONS OF THE GEOMETRIC APPROACH FOR SWITCHED LINEAR SYSTEMS}

The purpose of this section is to introduce the notions of the geometric approach useful to solve Problem 1. For the reader's convenience, some basic concepts are first reviewed [18], [19]. Then, new geometric notions are presented, like those of internal and external switched dynamics of a robust controlled invariant subspace and, above all, that of maximal robust controlled invariant subspace contained in a given subspace, with the property of being internally exponentially stabilizable over $\mathscr{S}_{\tau_{d}}$, for some finite positive real $\tau_{d}$. Indeed, such subspace will play a crucial role in the necessary and sufficient condition shown in the next section.

The definitions and properties reviewed below are referred to the generic $i$-th mode $\Sigma_{i}$ of the switched system $\Sigma_{\sigma(t)}$. Short notations for images and null spaces of input and output matrices, respectively, are used: $\mathcal{B}_{i}=\operatorname{im} B_{i}, \mathcal{H}_{i}=\operatorname{im} H_{i}$, and $\mathcal{E}_{i}=\operatorname{ker} E_{i}$, with $i \in \mathcal{I}$. A subspace $\mathcal{J} \subseteq \mathcal{X}$ is said to be an $A_{i}$-invariant subspace if $A_{i} \mathcal{J} \subseteq \mathcal{J}$. A subspace $\mathcal{V} \subseteq \mathcal{X}$ is said to be an $\left(A_{i}, \mathcal{B}_{i}\right)$-controlled invariant subspace if $A_{i} \mathcal{V} \subseteq \mathcal{V}+\mathcal{B}_{i}$. The subspace $\mathcal{V} \subseteq \mathcal{X}$ is an $\left(A_{i}, \mathcal{B}_{i}\right)$-controlled invariant subspace if and only if there exists a state feedback $F_{i}: \mathcal{X} \rightarrow \mathcal{U}$, such that $\left(A_{i}+B_{i} F_{i}\right) \mathcal{V} \subseteq \mathcal{V}$. The set of all $\left(A_{i}, \mathcal{B}_{i}\right)$-controlled invariant subspaces contained in a given subspace $\mathcal{K} \subseteq \mathcal{X}$ is an upper semilattice with respect to the sum and the inclusion of subspaces. The maximum of the semilattice is denoted by $\mathcal{V}^{*}(\mathcal{K})$ or by $\max \mathcal{V}\left(A_{i}, \mathcal{B}_{i}, \mathcal{K}\right)$ - the latter notation being adopted when the linear map $A_{i}$ and the subspace $\mathcal{B}_{i}$ need to be explicited. An algorithm for computing $\mathcal{V}^{*}(\mathcal{K})$ can be found in [19, Algorithm 4.1-2]. Moreover, an $\left(A_{i}, \mathcal{B}_{i}\right)$-controlled invariant subspace $\mathcal{V} \subseteq \mathcal{X}$ is said to be internally asymptotically stabilizable if there exists a state feedback $F_{i}$ such that $\mathcal{V}$ is an $\left(A_{i}+B_{i} F_{i}\right)$-invariant subspace and the restricted linear map $\left.\left(A_{i}+B_{i} F_{i}\right)\right|_{\mathcal{V}}$ is asymptotically stable. Likewise, $\mathcal{V}$ is said to be externally asymptotically stabilizable if there exists a state feedback $F_{i}$ such that $\mathcal{V}$ is an $\left(A_{i}+B_{i} F_{i}\right)$-invariant subspace and the induced linear map $\left.\left(A_{i}+B_{i} F_{i}\right)\right|_{\mathcal{X} / \mathcal{V}}$ is asymptotically stable. The set of all internally asymptotically stabilizable $\left(A_{i}, \mathcal{B}_{i}\right)$-controlled invariant subspaces contained in a given subspace $\mathcal{K} \subseteq \mathcal{X}$ has a maximal element, which is called the maximal $\operatorname{good}\left(\bar{A}_{i}, \mathcal{B}_{i}\right)$-controlled invariant subspace contained in $\mathcal{K}$ and is denoted by $\mathcal{V}_{g}^{*}(\mathcal{K})$ or by $\max \mathcal{V}_{g}\left(A_{i}, \mathcal{B}_{i}, \mathcal{K}\right)$. The subspace $\mathcal{V}_{g}^{*}(\mathcal{K})$ was introduced through a heuristic approach, including the computational algorithm [18, Section 5.6].

The definitions and properties revised above can be generalized to the set $\left\{\Sigma_{i}, i \in \mathcal{I}\right\}$ of the modes of the switched system $\Sigma_{\sigma(t)}$ by resorting to the notions of robust invariance and robust controlled invariance of a subspace, first introduced for a generic set of linear timeinvariant systems in [27]. A subspace $\mathcal{J}_{R} \subseteq \mathcal{X}$ is said to be a robust $A_{i}$-invariant subspace if it is an $A_{i}$-invariant subspace for all $i \in \mathcal{I}$. A subspace $\mathcal{V}_{R} \subseteq \mathcal{X}$ is said to be a robust $\left(A_{i}, \mathcal{B}_{i}\right)$-controlled invariant subspace if it is an $\left(A_{i}, \mathcal{B}_{i}\right)$-controlled invariant subspace for all $i \in \mathcal{I}$. Consequently, $\mathcal{V}_{R}$ is a robust $\left(A_{i}, \mathcal{B}_{i}\right)$-controlled invariant subspace if and only if there exists an indexed set of state feedbacks $\left\{F_{i}: \mathcal{X} \rightarrow \mathcal{U}, i \in \mathcal{I}\right\}$, such that $\left(A_{i}+B_{i} F_{i}\right) \mathcal{V}_{R} \subseteq \mathcal{V}_{R}$ for all $i \in \mathcal{I}$. The set of all robust $\left(A_{i}, \mathcal{B}_{i}\right)$-controlled invariant subspaces contained in a given subspace $\mathcal{K} \subseteq \mathcal{X}$ is an upper semilattice with respect to the sum and the inclusion. The maximum is denoted by $\mathcal{V}_{R}^{*}(\mathcal{K})$ or, equivalently, by $\max \mathcal{V}_{R}\left(A_{i}, \mathcal{B}_{i}, \mathcal{K}\right)$. A double recursion algorithm for computing $\mathcal{V}_{R}^{*}(\mathcal{K})$ was given in [27, Algorithm 1]. Since a robust $\left(A_{i}, \mathcal{B}_{i}\right)$-controlled invariant subspace $\mathcal{V}_{R}$ is an $\left(A_{i}, \mathcal{B}_{i}\right)$ controlled invariant subspace for each mode of the set $\left\{\Sigma_{i}, i \in \mathcal{I}\right\}$, the definitions of internal and external asymptotic stabilizability with respect to each mode apply to $\mathcal{V}_{R}$ in the same terms considered above. The check on internal and external asymptotic stabilizability of $\mathcal{V}_{R}$, with respect to each mode of the set $\left\{\Sigma_{i}, i \in \mathcal{I}\right\}$, can be done by distinguishing the assignable and the fixed internal and, respectively, external dynamics of $\mathcal{V}_{R}$ with respect to each mode. How to make such kind of distinctions is described in [25, Sections 3.1-3.2], though with reference to a specific robust controlled invariant subspace, 
which is the maximal one contained in $\mathcal{E}=\cap_{i \in \mathcal{I}} \mathcal{E}_{i}-$ i.e., $\mathcal{V}_{R}^{*}(\mathcal{E})$.

As to the maximal good $\left(A_{i}, \mathcal{B}_{i}\right)$-controlled invariant subspace contained in a given subspace, the following original, recursive construction shows how this notion extends to $\left\{\Sigma_{i}, i \in \mathcal{I}\right\}$, thus capturing robustness and stabilizability in one new geometric object.

Proposition 1: Consider the set $\left\{\Sigma_{i}, i \in \mathcal{I}\right\}$ of the modes of the switched linear system $\Sigma_{\sigma(t)}$ and a subspace $\mathcal{K} \subseteq \mathcal{X}$. The maximal robust $\left(A_{i}, \mathcal{B}_{i}\right)$-controlled invariant subspace contained in $\mathcal{K}$, with the property of being internally asymptotically stabilizable with respect to $\Sigma_{i}$ for all $i \in \mathcal{I}$ - henceforth denoted by $\mathcal{V}_{R, g}^{*}(\mathcal{K})$ - is the last term of the sequence $\mathcal{V}_{R}^{j}$, with $j=0,1, \ldots, \ell$, generated by the recursive algorithm

$$
\begin{aligned}
& \left\{\begin{aligned}
\mathcal{K}^{0} & =\mathcal{K}, \\
\mathcal{W}^{0} & =\bigcap_{i \in \mathcal{I}} \max \mathcal{V}_{g}\left(A_{i}, \mathcal{B}_{i}, \mathcal{K}^{0}\right), \\
\mathcal{V}_{R}^{0} & =\max \mathcal{V}_{R}\left(A_{i}, \mathcal{B}_{i}, \mathcal{W}^{0}\right),
\end{aligned}\right. \\
& \left\{\begin{aligned}
\mathcal{K}^{j} & =\mathcal{V}_{R}^{j-1}, \\
\mathcal{W}^{j} & =\bigcap_{i \in \mathcal{I}} \max \mathcal{V}_{g}\left(A_{i}, \mathcal{B}_{i}, \mathcal{K}^{j}\right), \quad j=1,2, \ldots, \ell, \\
\mathcal{V}_{R}^{j} & =\max \mathcal{V}_{R}\left(A_{i}, \mathcal{B}_{i}, \mathcal{W}^{j}\right),
\end{aligned}\right.
\end{aligned}
$$

where $\ell$, with $0 \leq \ell \leq \operatorname{dim} \mathcal{K}$, is the least integer such that $\mathcal{V}_{R}^{\ell}$ is internally asymptotically stabilizable with respect to $\Sigma_{i}$ for all $i \in \mathcal{I}$.

Proof: First, note that the sequence of subspaces $\mathcal{V}_{R}^{j}$, with $j=0,1, \ldots, \ell$, is monotone nonincreasing (i.e., $\mathcal{V}_{R}^{j+1} \subseteq \mathcal{V}_{R}^{j}$ for all $j=0,1, \ldots, \ell-1)$ and it converges in $\ell=\operatorname{dim} \mathcal{K}$ steps at most, the worst case occurring when $\mathcal{V}_{R, g}^{*}(\mathcal{K})=\{0\}$ and the dimension of the subspaces subsequently generated decreases by one at each iteration. Also note that $\mathcal{V}_{R, g}^{*}(\mathcal{K})$ is a robust $\left(A_{i}, \mathcal{B}_{i}\right)$-controlled invariant subspace, with the property of being internally asymptotically stabilizable with respect to $\Sigma_{i}$ for all $i \in \mathcal{I}$ and that of being contained in $\mathcal{K}$, by construction. Then, in order to prove maximality of $\mathcal{V}_{R, g}^{*}(\mathcal{K})$, a generic robust $\left(A_{i}, \mathcal{B}_{i}\right)$-controlled invariant subspace $\mathcal{V} \subseteq \mathcal{K}$, with the property of being internally stabilizable with respect to $\Sigma_{i}$ for all $i \in \mathcal{I}$, is considered and shown to be contained in $\mathcal{V}_{R, g}^{*}(\mathcal{K})$. The subspace $\mathcal{V}$, as an internally asymptotically stabilizable $\left(A_{i}, \mathcal{B}_{i}\right)$-controlled invariant subspace for all $i \in \mathcal{I}$, with the property of being contained in $\mathcal{K}$, is contained in $\max \mathcal{V}_{g}\left(A_{i}, \mathcal{B}_{i}, \mathcal{K}^{0}\right)$ for all $i \in \mathcal{I}$, which implies that $\mathcal{V} \subseteq \mathcal{W}^{0}$. Moreover, $\mathcal{V}$, as a robust $\left(A_{i}, \mathcal{B}_{i}\right)$-controlled invariant subspace contained in $\mathcal{W}^{0}$ is contained in $\max \mathcal{V}_{R}\left(A_{i}, \mathcal{B}_{i}, \mathcal{W}^{0}\right)$, by the maximality of the latter. Then, by iterating this reasoning, one gets that $\mathcal{V} \subseteq \mathcal{V}_{R}^{j}$ for all $j=0,1, \ldots, \ell$, which implies $\mathcal{V} \subseteq \mathcal{V}_{R, g}^{*}(\mathcal{K})$.

Definition 1: The maximal robust $\left(A_{i}, \mathcal{B}_{i}\right)$-controlled invariant subspace contained in $\mathcal{K}$, with the property of being internally asymptotically stabilizable with respect to all modes of the set $\left\{\Sigma_{i}, i \in \mathcal{I}\right\}$ - namely, the subspace $\mathcal{V}_{R, g}^{*}(\mathcal{K})$ - is called the maximal good robust controlled invariant subspace contained in $\mathcal{K}$ for the set $\left\{\Sigma_{i}, i \in \mathcal{I}\right\}$.

Concerning the definition of the subspace $\mathcal{V}_{R, g}^{*}(\mathcal{K})$ and the related recursive algorithm - i.e., Definition 1 and Proposition 1, the following aspects are worth being highlighted.

Remark 1: The notion of maximal good robust controlled invariant subspace contained in $\mathcal{K}$ captures at one time the feature of robust controlled invariance and that of internal asymptotic stabilizability with respect to each mode of the switched linear system. This peculiarity is clearly pointed out by the fact that the construction of $\mathcal{V}_{R, g}^{*}(\mathcal{K})$ proceeds with the iteration of two stages, which respectively involve internal stabilizability with respect to each mode $\Sigma_{i}$ (construction of the subspaces $\max \mathcal{V}_{g}\left(A_{i}, \mathcal{B}_{i}, \mathcal{K}^{j}\right)$ ) and robust controlled invariance with respect to the set $\left\{\Sigma_{i}, i \in \mathcal{I}\right\}$ (construction of the subspaces $\max \mathcal{V}_{R}\left(A_{i}, \mathcal{B}_{i}, \mathcal{W}^{j}\right)$ ). As will be shown in the remainder of this work, this double nature of the subspace $\mathcal{V}_{R, g}^{*}(\mathcal{K})$ is crucial when dealing with switched systems, to the extent that a special subspace of this kind - namely $\mathcal{V}_{R, g}^{*}(\mathcal{E})$, where $\mathcal{E}=\cap_{i \in \mathcal{I}} \mathcal{E}_{i}$ will play a key role in the necessary and sufficient condition to solve Problem 1.

Remark 2: From the computational point of view, it is relevant that the recursive algorithm of Proposition 1 can be implemented in Matlab by using software specifically developed for the geometric approach [28]. In particular, the Matlab functions vstarg and robcoin can respectively be used to compute the subspaces $\max \mathcal{V}_{g}\left(A_{i}, \mathcal{B}_{i}, \mathcal{K}^{j}\right)$ and $\max \mathcal{V}_{R}\left(A_{i}, \mathcal{B}_{i}, \mathcal{W}^{j}\right)$, while stabv is an effective means to check on internal and external stabilizability of a controlled invariant subspace.

Remark 3: An interesting variant of the algorithm of Proposition 1 is presented in Appendix, where the algorithm is modified in such a way that the generated subspace is the maximal robust controlled invariant subspace contained in $\mathcal{K}$, whose internal dynamics is assignable with respect to each mode of the set $\left\{\Sigma_{i}, i \in \mathcal{I}\right\}$.

The remainder of this section is aimed at establishing the needed relations between the properties of robustness and stabilizability considered above and the property of exponential stabilizability over $\mathscr{S}_{\tau_{d}}$, for some positive real $\tau_{d}$, which applies to switched dynamics.

The definitions and properties considered above with reference to the set of the modes $\left\{\Sigma_{i}, i \in \mathcal{I}\right\}$ can directly be referred to the switched linear system $\Sigma_{\sigma(t)}$, so that it makes sense to refer to a robust invariant subspace or to a robust controlled invariant subspace for $\Sigma_{\sigma(t)}$. In particular, the characterization of a robust controlled invariant subspace $\mathcal{V}_{R}$ for $\Sigma_{\sigma(t)}$ can be expressed in terms of the existence of a switched state feedback $F_{\sigma(t)}$, such that $\mathcal{V}_{R}$ is a robust invariant subspace for the closed-loop system $\Sigma_{F, \sigma(t)}$. Such switched state feedback $F_{\sigma(t)}$ is called a friend of $\mathcal{V}_{R}$. In this framework, the definitions of internal and external switched dynamics associated to $\mathcal{V}_{R}$ by $F_{\sigma(t)}$ are stated as follows.

Definition 2: Let $\mathcal{V}_{R}$ be a robust controlled invariant subspace for the switched linear system $\Sigma_{\sigma(t)}$ and let the switched state feedback $F_{\sigma(t)}$ be a friend of $\mathcal{V}_{R}$ :

i) the switched dynamics $\left(A_{\sigma(t)}+B_{\sigma(t)} F_{\sigma(t)}\right) \mid \mathcal{V}_{R}$ is called the internal switched dynamics associated to $\mathcal{V}_{R}$ by $F_{\sigma(t)}$;

ii) the switched dynamics $\left.\left(A_{\sigma(t)}+B_{\sigma(t)} F_{\sigma(t)}\right)\right|_{\mathcal{X} / \mathcal{V}_{R}}$ is called the external switched dynamics associated to $\mathcal{V}_{R}$ by $F_{\sigma(t)}$.

Hence, the definitions of internal and external stabilizability over $\mathscr{S}_{\tau_{d}}$ of a robust controlled invariant subspace $\mathcal{V}_{R}$ for the switched linear system $\Sigma_{\sigma(t)}$ are stated as follows. A characterization of each property is given next.

Definition 3: A robust controlled invariant subspace $\mathcal{V}_{R}$ for the switched linear system $\Sigma_{\sigma(t)}$ is said to be

i) internally exponentially stabilizable over $\mathscr{S}_{\tau_{d}}$ if there exists a friend $F_{\sigma(t)}$ of $\mathcal{V}_{R}$, such that $\left.\left(A_{\sigma(t)}+B_{\sigma(t)} F_{\sigma(t)}\right)\right|_{\mathcal{V}_{R}}$ is exponentially stable over $\mathscr{S}_{\tau_{d}}$;

ii) externally exponentially stabilizable over $\mathscr{S}_{\tau_{d}}$ if there exists a friend $F_{\sigma(t)}$ of $\mathcal{V}_{R}$, such that $\left.\left(A_{\sigma(t)}+B_{\sigma(t)} F_{\sigma(t)}\right)\right|_{\mathcal{X} / \mathcal{V}_{R}}$ is exponentially stable over $\mathscr{S}_{\tau_{d}}$.

Proposition 2: Let $\mathcal{V}_{R}$ be a robust controlled invariant subspace for the switched linear system $\Sigma_{\sigma(t)}$. Hence,

i) $\mathcal{V}_{R}$ is internally exponentially stabilizable over $\mathscr{S}_{\tau_{d}}$, for some positive real $\tau_{d}$, if and only if there exists a friend $F_{\sigma(t)}$, associated to the set $\left\{F_{i}, i \in \mathcal{I}\right\}$, such that the restricted maps of the set $\left\{\left(A_{i}+B_{i} F_{i}\right) \mid \mathcal{V}_{R}, i \in \mathcal{I}\right\}$ are asymptotically stable for all $i \in \mathcal{I}$;

ii) $\mathcal{V}_{R}$ is externally exponentially stabilizable over $\mathscr{S}_{\tau_{d}}$, for some positive real $\tau_{d}$, if and only if there exists a friend $F_{\sigma(t)}$, associated to the set $\left\{F_{i}, i \in \mathcal{I}\right\}$, such that the induced maps of the set $\left\{\left.\left(A_{i}+B_{i} F_{i}\right)\right|_{\mathcal{X} / \mathcal{V}_{R}}, i \in \mathcal{I}\right\}$ are asymptotically stable for all $i \in \mathcal{I}$. 
Proof: The if-part of the statements is a consequence of [17, Lemma 2]. The converse follows from the fact that, for all $i \in \mathcal{I}$, the switching signal $\sigma(t)=i$ for all $t \in \mathbb{R}^{+}$belongs to $\mathcal{S}_{\tau_{d}}$ for any positive real $\tau_{d}$.

Remark 4: Proposition 2 explicitly states that, when the positive real $\tau_{d}$ is not a-priori fixed, but can be picked sufficiently large (depending on the modes of the considered switched dynamics as shown, e.g., in [17]) — which is the case dealt with in this work, differently from other literature [29], where $\tau_{d}$ is given then, exponential stabilizability over $\mathscr{S}_{\tau_{d}}$ is equivalent to asymptotic stabilizability of each mode of the switched dynamics. Obviously, this equivalence holds for any considered switched dynamics and the associated modes.

Remark 5: As a noticeable consequence of Proposition 2, the subspace $\mathcal{V}_{R, g}^{*}(\mathcal{K})$, introduced in Definition 1, is also the maximal robust controlled invariant subspace for $\Sigma_{\sigma(t)}$, contained in $\mathcal{K}$ and enjoying the property of being internally exponentially stabilizable over $\mathscr{S}_{\tau_{d}}$, for some positive real $\tau_{d}$.

\section{Problem Solution}

In this section, the necessary and sufficient condition for solving Problem 1 is stated. Some propositions, functional to the proof of the main result, are premised. A crucial role in the necessary and sufficient condition is played by the subspace $\mathcal{V}_{R, g}^{*}(\mathcal{E})$, where $\mathcal{E}=\cap_{i \in \mathcal{I}} \mathcal{E}_{i}$. Since $\mathcal{V}_{R, g}^{*}(\mathcal{E})$ is the only maximal internally stabilizable robust controlled invariant subspace considered henceforth, the shorter notation $\mathcal{V}_{R, g}^{*}$ is adopted.

Proposition 3: Let the switched linear system $\Sigma_{\sigma(t)}$ be exponentially stabilizable over $\mathscr{S}_{\tau_{d}}$. Then, any robust controlled invariant subspace $\mathcal{V}_{R} \subseteq \mathcal{X}$ for $\Sigma_{\sigma(t)}$ is externally exponentially stabilizable over $\mathscr{S}_{\tau_{d}}$. Let $\mathcal{V}_{R}$ be a robust controlled invariant subspace for $\Sigma_{\sigma(t)}$, both internally and externally exponentially stabilizable over $\mathscr{S}_{\tau_{d}}$, then $\Sigma_{\sigma(t)}$ is exponentially stabilizable over $\mathscr{S}_{\tau_{d}}$.

Proof: It ensues from Proposition 2, in light of [19, Property 4.1-16].

Proposition 4: Let $\mathcal{V}_{R} \subseteq \mathcal{X}$ be a robust controlled invariant subspace for the switched linear system $\Sigma_{\sigma(t)}$, with the properties of being externally exponentially stabilizable over $\mathscr{S}_{\tau_{d}^{\prime}}$ and internally exponentially stabilizable over $\mathscr{S}_{\tau_{d}^{\prime \prime}}$, with $\tau_{d}^{\prime \prime} \neq \tau_{d}^{\prime}$. Then, there exists a friend $F_{\sigma(t)}$ of $\mathcal{V}_{R}$, such that $\left.\left(A_{\sigma(t)}+B_{\sigma(t)} F_{\sigma(t)}\right)\right|_{\mathcal{V}_{R}}$ and $\left.\left(A_{\sigma(t)}+B_{\sigma(t)} F_{\sigma(t)}\right)\right|_{\mathcal{X} / \mathcal{V}_{R}}$ are both exponentially stable over $\mathscr{S}_{\tau_{d}}$, for some positive real $\tau_{d}$.

Proof: It follows from Proposition 2, with $\tau_{d} \geq \max \left\{\tau_{d}^{\prime}, \tau_{d}^{\prime \prime}\right\}$.

Lemma 1: Consider the switched linear system $\Sigma_{\sigma(t)}$, with the modes $\left\{\Sigma_{i}, i \in \mathcal{I}\right\}$. Consider the subspace $\mathcal{V}_{R, g}^{*}$. Let the switched state feedback $F_{\sigma(t)}$, associated with the indexed set $\left\{F_{i}, i \in \mathcal{I}\right\}$, be a friend of $\mathcal{V}_{R, g}^{*}$. Let $\mathcal{H}_{i} \subseteq \mathcal{V}_{R, g}^{*}$, for all $i \in \mathcal{I}$. Consider the closedloop switched linear system $\Sigma_{F, \sigma(t)}$, with the modes $\left\{\Sigma_{F, i}, i \in \mathcal{I}\right\}$. Perform the state-space basis transformation $T=\left[\begin{array}{ll}T_{1} & T_{2}\end{array}\right]$, where $\operatorname{im} T_{1}=\mathcal{V}_{R, g}^{*}$. Then, with respect to new coordinates,

$$
\begin{aligned}
A_{F, i}^{\prime}= & T^{-1}\left(A_{i}+B_{i} F_{i}\right) T= \\
& {\left[\begin{array}{cc}
A_{11, i}^{\prime}+B_{1, i}^{\prime} F_{1, i}^{\prime} & A_{12, i}^{\prime}+B_{1, i}^{\prime} F_{2, i}^{\prime} \\
O & A_{22, i}^{\prime}+B_{2, i}^{\prime} F_{2, i}^{\prime}
\end{array}\right], } \\
H_{i}^{\prime}= & T^{-1} H_{i}=\left[\begin{array}{c}
H_{1, i}^{\prime} \\
O
\end{array}\right], \\
E_{i}^{\prime}= & E_{i} T=\left[\begin{array}{ll}
O & E_{2, i}^{\prime}
\end{array}\right],
\end{aligned}
$$

for all $i \in \mathcal{I}$.

Proof: First, note that, with respect to the new coordinates, $\mathcal{V}_{R, g}^{*}=\operatorname{im}\left(T^{-1} T_{1}\right)=\left[\begin{array}{ll}I & O\end{array}\right]^{\top}$. Hence, the zero block in (5), for all $i \in \mathcal{I}$, is due to $\mathcal{V}_{R, g}^{*}$ being a robust $\left(A_{i}+B_{i} F_{i}\right)$-invariant subspace. The zero block in (6), for all $i \in \mathcal{I}$, is due to $\mathcal{H}_{i} \subseteq \mathcal{V}_{R, g}^{*}$, for all $i \in \mathcal{I}$.
The zero block in (7) is due to $\mathcal{V}_{R, g}^{*} \subseteq \mathcal{E}$, which implies $\mathcal{V}_{R, g}^{*} \subseteq \mathcal{E}_{i}$, for all $i \in \mathcal{I}$.

Theorem 1: Consider the switched linear system $\Sigma_{\sigma(t)}$. Let the modes $\left\{\Sigma_{i}, i \in \mathcal{I}\right\}$ be asymptotically stabilizable. Consider the subspace $\mathcal{V}_{R, g}^{*}$ and the images $\mathcal{H}_{i}$ of the disturbance input matrices $H_{i}$, with $i \in \mathcal{I}$. Then, Problem 1 has a solution if and only if

$$
\mathcal{H}_{i} \subseteq \mathcal{V}_{R, g}^{*}, \quad \forall i \in \mathcal{I} .
$$

Proof: If. First, note that, since the modes $\left\{\Sigma_{i}, i \in \mathcal{I}\right\}$ are asymptotically stabilizable, there exists a positive constant $\tau_{d}$ such that $\Sigma_{\sigma(t)}$ is exponentially stabilizable over $\mathscr{S}_{\tau_{d}}$ [17, Lemma 2]. Hence, by virtue of Propositions 3 and 4, there exists a friend $F_{\sigma(t)}$ of $\mathcal{V}_{R, g}^{*}$, such that $\left.\left(A_{\sigma(t)}+B_{\sigma(t)} F_{\sigma(t)}\right)\right|_{\mathcal{V}_{R, g}^{*}}$ and $\left.\left(A_{\sigma(t)}+B_{\sigma(t)} F_{\sigma(t)}\right)\right|_{\mathcal{X} / \mathcal{V}_{R, g}^{*}}$ are exponentially stable over $\mathscr{S}_{\tilde{\tau}_{d}}$, with $\tilde{\tau}_{d} \geq \tau_{d}$. Then, it will be shown that the closed-loop switched system $\Sigma_{F, \sigma(t)}$, obtained by applying to $\Sigma_{\sigma(t)}$ such switched state feedback $F_{\sigma(t)}$, satisfies Requirements $\mathcal{R} 1$ and $\mathcal{R} 2$ of Problem 1 . In order to show that Requirement $\mathcal{R} 1$ is met, let us refer to the coordinates introduced in Lemma 1 and consider the state $x(t)=\left[x_{1}(t)^{\top} x_{2}(t)^{\top}\right]^{\top}$, with $t \in \mathbb{R}^{+}$, consistently partitioned. Hence, the modes $\left\{\Sigma_{F, i}, i \in \mathcal{I}\right\}$ of $\Sigma_{F, \sigma(t)}$ are described by

$$
\Sigma_{F, i} \equiv\left\{\begin{aligned}
\dot{x}_{1}(t)= & \left(A_{11, i}^{\prime}+B_{1, i}^{\prime} F_{1, i}^{\prime}\right) x_{1}(t)+H_{1, i}^{\prime} h(t) \\
& +\left(A_{12, i}^{\prime}+B_{1, i}^{\prime} F_{2, i}^{\prime}\right) x_{2}(t), \quad i \in \mathcal{I}, \\
\dot{x}_{2}(t)= & \left(A_{22, i}^{\prime}+B_{2, i}^{\prime} F_{2, i}^{\prime}\right) x_{2}(t), \\
e(t)= & E_{2, i}^{\prime} x_{2}(t),
\end{aligned}\right.
$$

where (5)-(8) have been taken into account. In light of (9), the assumption $x(0)=0$, implies $x_{2}(t)=0$, for all $t \in \mathbb{R}^{+}$, which, in turn, implies $e(t)=0$, for all $t \in \mathbb{R}^{+}$, for any admissible input signal $h(t)$, with $t \in \mathbb{R}^{+}$. Note that these considerations hold for all $i \in \mathcal{I}$, which means that the disturbance input is structurally decoupled. In order to prove that Requirement $\mathcal{R} 2$ is also met, note that the dynamic matrix in (9) - i.e., $A_{F, i}^{\prime}$ in $(5)-$ has an upper block-triangular structure for all $i \in \mathcal{I}$. This property and the special choice of $F_{\sigma(t)}$ guarantee that the switched dynamics $A_{F, \sigma(t)}$, associated with the set $\left\{A_{F, i}^{\prime}, i \in \mathcal{I}\right\}$, is exponentially stable over $\mathscr{S}_{\tilde{\tau}_{d}}$, or, equivalently, that the dynamics of the set $\left\{A_{F, i}^{\prime}, i \in \mathcal{I}\right\}$ are asymptotically stable.

Only if. Let Problem 1 have a solution. Then, there exists a switched state feedback $F_{\sigma(t)}$, such that the closed-loop switched system $\Sigma_{F, \sigma(t)}$ satisfies Requirements $\mathcal{R} 1$ and $\mathcal{R} 2$ of Problem 1 . This implies that, for all $i \in \mathcal{I}, \mathcal{H}_{i}$ is contained in the maximal robust invariant subspace contained in $\mathcal{E}$ for $\Sigma_{F, \sigma(t)}$ and that $\Sigma_{F, \sigma(t)}$ is exponentially stable over $\mathscr{S}_{\tau_{d}}$, for some $\tau_{d}$. Hence, $\mathcal{V}$ is a robust controlled invariant subspace contained in $\mathcal{E}$ for $\Sigma_{\sigma(t)}$. Moreover, exponential stability of $\Sigma_{F, \sigma(t)}$ over $\mathscr{S}_{\tau_{d}}$ implies, in particular, that $\mathcal{V}$ is internally exponentially stabilizable over $\mathscr{S}_{\tau_{d}}$. Therefore, $\mathcal{V} \subseteq \mathcal{V}_{R, g}^{*}$, by maximality of the latter, and the conclusion follows.

Remark 6: The if-part of the proof of Theorem 1 is constructive: i.e., it shows how to choose the switched state feedback achieving decoupling with stability, under the given condition.

Remark 7: Even if the initial state is not the origin, then identically zero output is still guaranteed, provided that the initial state belongs to the subspace $\mathcal{V}_{R, g}^{*}$. In fact, if the initial state belongs to $\mathcal{V}_{R, g}^{*}$, then $x_{2}(0)=0$. Hence, $x_{2}(t)=0$, for all $t \in \mathbb{R}^{+}$, which implies $e(t)=0$, for all $t \in \mathbb{R}^{+}$, for any admissible input signal $h(t)$, with $t \in \mathbb{R}^{+}$.

\section{An Illustrative EXAMPLE}

This section aims at illustrating the steps of the synthesis procedure discussed so far, through a numerical example. The computational aids consist of the Matlab Control System Toolbox and the Geometric Approach Toolbox [28]. The variables are displayed in scaled fixed 
point format with five digits, although calculations are made in the appropriate floating point precision. Consider the switched system $\Sigma_{\sigma(t)}$, defined by (1), with the modes $\left\{\Sigma_{i}, i \in \mathcal{I}\right\}$, defined by (2). Let $\mathcal{I}=\{1,2\}$ and

$$
\begin{aligned}
& A_{1}= \\
& {\left[\begin{array}{cccccccc}
-5.8 & 0 & 0 & 0 & 0 & 0 & 0 & 0 \\
10 & -4 & 0 & 0 & 0 & 0 & 0 & 0 \\
0 & 0 & -0.2 & 0 & -10.4 & 0 & 7.6 & 2.2 \\
0 & 0 & 0 & -1 & 0 & 5 & 0 & 0 \\
0 & 0 & 0 & 0 & -7.3 & 0 & 0 & 5.5 \\
0 & 0 & 0 & 0 & 0 & -1 & 0 & 0 \\
0 & 0 & -10.1 & 0 & -9.9 & 0 & -25.5 & 4.2 \\
0 & 0 & 0 & 0 & 0 & 0 & 0 & 1
\end{array}\right],} \\
& A_{2}= \\
& {\left[\begin{array}{cccccccc}
-10.5 & 0 & -0.2 & 0 & 1.8 & 0 & -0.2 & 0 \\
0.3 & -13.9 & 6.8 & 0 & -25.5 & 0 & 3.2 & -3.1 \\
0 & 0 & -4.4 & 0 & -15.1 & 0 & 6.7 & 0 \\
0 & 0 & 0 & -1 & 0 & 0 & 0 & 0 \\
0 & 0 & 2 & 0 & -80 & 0 & 2.2 & 0 \\
0 & 0 & 0 & -3 & 0 & -1 & 0 & 0 \\
0 & 0 & -7.8 & 0 & 10.1 & 0 & -20.6 & 0 \\
2.5 & 0 & 3.4 & 0 & -1 & 0 & -1.8 & -23
\end{array}\right] \text {, }} \\
& B_{1}= \\
& {\left[\begin{array}{ccc}
0 & 60 & 0 \\
0 & -50 & 0 \\
1.9 & 4.8 & 0 \\
0 & 0 & 0 \\
-8.8 & 0 & -7.1 \\
0 & 0 & 0 \\
-2 & 3.5 & -17.7 \\
7.6 & 0 & 0
\end{array}\right]} \\
& H_{1}= \\
& {\left[\begin{array}{ccc}
0 & 0 & 0 \\
0 & 0 & 0 \\
0 & -3 & -0.3 \\
0 & 0 & 0 \\
2.1 & 0 & 0 \\
0 & 0 & 0 \\
0 & 0 & 0 \\
0 & 1 & 0
\end{array}\right]} \\
& E_{1}=\left[\begin{array}{cccccccc}
0 & 0 & 0 & 0 & 0 & 0 & -3 & 0 \\
0 & -1 & 0 & 0 & 0 & 0 & 0 & 0 \\
0 & 0 & 0 & -1 & 0 & 0 & 0 & 0 \\
0 & 0 & 0 & 0 & 0 & 1 & 0 & 0
\end{array}\right] \text {, } \\
& E_{2}=\left[\begin{array}{cccccccc}
0 & 0 & 0 & 0 & 0 & 0 & -15 & 0 \\
5 & -5 & 0 & 0 & 0 & 0 & 0 & 0 \\
0 & 0 & 0 & -1 & 0 & 0 & 0 & 0 \\
0 & 0 & 0 & 0 & 0 & 1 & 0 & 0
\end{array}\right] \text {, } \\
& {\left[\begin{array}{ccc}
0.1 & 1.7 & 1.6 \\
-1.6 & -3.7 & 0.2 \\
-1 & -1 & 0.5 \\
0 & 0 & 0 \\
-2 & -2 & 0.2 \\
0 & 0 & 0 \\
0.7 & 0.7 & -2.3 \\
0.5 & -2.2 & -0.3
\end{array}\right]} \\
& \mathrm{H}_{2}= \\
& {\left[\begin{array}{ccc}
0 & 0 & 0 \\
0 & 0 & 0 \\
0 & 0 & -2 \\
0 & 0 & 0 \\
2 & 0 & 0 \\
0 & 0 & 0 \\
0 & 0 & 0 \\
0.5 & 0.25 & 0
\end{array}\right]}
\end{aligned}
$$

With regard to the given switched system, it is worth noting that the mode $\Sigma_{1}$ is not asymptotically stable, since $A_{1}$ has one eigenvalue at 1 , but it is stabilizable, since its reachable subspace is externally stable. Moreover, $\Sigma_{1}$ is not minimum-phase, since it has one zero at 6.2 . As to the mode $\Sigma_{2}$, this is both asymptotically stable and minimum-phase. By applying the recursive algorithm of Proposition 1, one gets that the maximal internally stabilizable robust $\left(A_{i}, \mathcal{B}_{i}\right)$-controlled invariant subspace contained in $\mathcal{E}=\mathcal{E}_{1} \cap \mathcal{E}_{2}$ namely, the subspace $\mathcal{V}_{R, g}^{*}$ - is given by

$$
\mathcal{V}_{R, g}^{*}=\operatorname{im}\left[\begin{array}{ccc}
0 & 0 & 0 \\
0 & 0 & 0 \\
1 & 0 & 0 \\
0 & 0 & 0 \\
0 & -1 & 0 \\
0 & 0 & 0 \\
0 & 0 & 0 \\
0 & 0 & 1
\end{array}\right]
$$

The inclusions $\mathcal{H}_{i} \subseteq \mathcal{V}_{R, g}^{*}$ hold for all $i \in \mathcal{I}$, as can be checked by comparing the basis matrix of the subspace $\mathcal{V}_{R, g}^{*}$ with $H_{1}$ and $H_{2}$ (basis matrices of $\mathcal{H}_{1}$ and $\mathcal{H}_{2}$, respectively). Hence, Problem 1 has a solution, according to Theorem 1. A set of state feedbacks $\left\{F_{i}, i \in \mathcal{I}\right\}$ that guarantees state-feedback invariance along with internal and external asymptotic stability of $\mathcal{V}_{R, g}^{*}$ with respect to each mode is given by

$$
\begin{aligned}
& F_{1}=\left[\begin{array}{cccccc}
0.0008 & 0.0002 & 0.2473 & 0 & 0.1940 & 0 \\
0.0918 & -0.0059 & 0 & 0 & 0 & 0 \\
0.0066 & 0.0018 & -0.5986 & 0 & -0.5812 & 0 \\
& & & & -0.1481 & 0.0803 \\
& & & & 0.0001 & 0 \\
& & & -1.3109 & 0.2282
\end{array}\right] \\
& F_{2}=\left[\begin{array}{cccccc}
-13.8084 & -14.9010 & -5.7610 & 0 & -17.6645 & 0 \\
5.9962 & 3.6810 & 4.1188 & 0 & 0.7052 & 0 \\
-2.2195 & -3.1276 & -3.8911 & 0 & -0.7702 & 0
\end{array}\right. \\
& \left.\begin{array}{cc}
-26.1586 & -5.5118 \\
11.7625 & 1.4793 \\
-10.9650 & -1.2273
\end{array}\right]
\end{aligned}
$$

The set of the eigenvalues of the internal dynamics of $\mathcal{V}_{R, g}^{*}$ with respect to each mode respectively are $\mathscr{L}_{i, 1}=\{-0.1,-0.2,-0.3\}$ and $\mathscr{L}_{i, 2}=\{-5.8166,-34.2142,-39.55\}$. It is worth mentioning that the former set, $\mathscr{L}_{i, 1}$, has been completely assigned through $F_{1}$, while the latter, $\mathscr{L}_{i, 2}$, is fixed - i.e., it does not depend on $F_{2}$. The set of the eigenvalues of the external dynamics of $\mathcal{V}_{R, g}^{*}$ with respect to each mode respectively are $\mathscr{L}_{e, 1}=\{-1,-2,-3,-1,-1\}$ and $\mathscr{L}_{e, 2}=\{-4,-5,-6,-1,-1\}$. The former three eigenvalues of each set, $\mathscr{L}_{e, 1}$ and $\mathscr{L}_{e, 2}$, have been assigned by $F_{1}$ and $F_{2}$, respectively. Instead, the last two eigenvalues of each set are fixed. Hence, the linear maps $F_{1}$ and $F_{2}$ given above solves Problem 1, as shown in the if-part of the proof of Theorem 1 .

In particular, as to stability of the closed-loop switched dynamics, it is worth noting that both modes, $\Sigma_{F, 1}$ and $\Sigma_{F, 2}$, of the closed-loop switched system $\Sigma_{F, \sigma(t)}$ are asymptotically stable. In fact, the set of the eigenvalues of $A_{F, 1}$ is given by $\mathscr{L}_{i, 1} \uplus \mathscr{L}_{e, 1}$, while that of $A_{F, 2}$ is given by $\mathscr{L}_{i, 2} \uplus \mathscr{L}_{e, 2}$. However, the closed-loop switched system is not exponentially stable under arbitrary switching. For instance, it is easy to show that periodic switching between the two modes with a dwell-time $\tau_{h p}=1 \mathrm{~s}$ in each mode generates instability of the switched system. In fact, the state transition matrix over one period - i.e., $\Phi\left(2 \tau_{h p}\right)=e^{A_{F, 2} \tau_{h p}} e^{A_{F, 1} \tau_{h p}}-$ has one eigenvalue at -1.7489 , that is outside the open unit disc of the complex plane. Nonetheless, by virtue of [17, Lemma 2], there exists a a sufficiently large $\tau_{d}$, such that the closed-loop dynamics is exponentially stable over $\mathscr{S}_{\tau_{d}}$. For instance, by applying the procedure described in the abovementioned lemma, one can find $\tau_{d}=13.1 \mathrm{~s}$.

\section{Vi. Conclusions}

A necessary and sufficient condition for the solution of the disturbance decoupling problem with exponential stability in switched linear systems subject to switching signals with a sufficiently large dwell-time has been shown. A complete algorithmic framework for the synthesis of the compensation scheme has been outlined. An illustrative example has been worked out with the twofold aim of describing the single steps of the synthesis procedure and highlighting the effectiveness of the devised synthesis procedure. The methodological background consists of both classic and novel concepts of the geometric approach, enhanced with notions specifically oriented to switched linear systems. In particular, the hybrid nature of switched linear systems is caught by the so-called maximal good robust controlled invariant subspace contained in the null space of the output 
of the to-be-controlled switched system: this subspace is the one that plays the key role in the abovementioned necessary and sufficient condition.

\section{APPENDIX}

First, the notion of controllability subspace for the mode $\Sigma_{i}$ is reviewed [18], [19]. A subspace $\mathcal{R} \subseteq \mathcal{X}$ is said to be a controllability subspace for $\Sigma_{i}$ if there exists a state feedback $F_{i}: \mathcal{X} \rightarrow \mathcal{U}$, such that $\mathcal{R}=\left\langle A_{i}+B_{i} F_{i} \mid \mathcal{B}_{i} \cap \mathcal{R}\right\rangle$, where the notation $\langle A, \mathcal{B}\rangle$ stands for the minimal $A$-invariant subspace containing $\mathcal{B}$. Moreover, if $\mathcal{V}$ is an $\left(A_{i}, \mathcal{B}_{i}\right)$-controlled invariant subspace for $\Sigma_{i}$, the maximal controllability subspace contained in $\mathcal{V}$, denoted by $\mathcal{R}^{*}(\mathcal{V})$ or by $\max \mathcal{R}\left(A_{i}, \mathcal{B}_{i}, \mathcal{V}\right)$, is such that the internal dynamics of $\mathcal{R}^{*}(\mathcal{V})$ coincides with that part of the internal dynamics of $\mathcal{V}$ assignable by a friend of $\mathcal{V}$ (which, as can be shown, is also a friend of $\mathcal{R}^{*}(\mathcal{V})-$ see, e.g., $\left[19\right.$, Chapter 4]). This notion extends to the set $\left\{\Sigma_{i}, i \in \mathcal{I}\right\}$ of the modes of $\Sigma_{\sigma(t)}$ as described below.

Proposition 5: Consider the set $\left\{\Sigma_{i}, i \in \mathcal{I}\right\}$ of the modes of the switched linear system $\Sigma_{\sigma(t)}$ and a subspace $\mathcal{K} \subseteq \mathcal{X}$. The maximal robust $\left(A_{i}, \mathcal{B}_{i}\right)$-controlled invariant subspace contained in $\mathcal{K}$, with the property of being a controllability subspace with respect to $\Sigma_{i}$ for all $i \in \mathcal{I}$ - henceforth denoted by $\mathcal{R}_{R}^{*}(\mathcal{K})$ - is the last term of the sequence $\mathcal{R}_{R}^{j}$, with $j=0,1, \ldots, \ell$, generated by the recursive algorithm

$$
\begin{aligned}
& \left\{\begin{aligned}
\mathcal{K}^{0} & =\mathcal{K} \\
\mathcal{W}^{0} & =\bigcap_{i \in \mathcal{I}} \max \mathcal{R}\left(A_{i}, \mathcal{B}_{i}, \mathcal{K}^{0}\right), \\
\mathcal{R}_{R}^{0} & =\max \mathcal{V}_{R}\left(A_{i}, \mathcal{B}_{i}, \mathcal{W}^{0}\right),
\end{aligned}\right. \\
& \left\{\begin{aligned}
\mathcal{K}^{j} & =\mathcal{V}_{R}^{j-1} \\
\mathcal{W}^{j} & =\bigcap_{i \in \mathcal{I}} \max \mathcal{R}\left(A_{i}, \mathcal{B}_{i}, \mathcal{K}^{j}\right), \quad j=1,2, \ldots, \ell, \\
\mathcal{R}_{R}^{j} & =\max \mathcal{V}_{R}\left(A_{i}, \mathcal{B}_{i}, \mathcal{W}^{j}\right),
\end{aligned}\right.
\end{aligned}
$$

where $\ell$, with $0 \leq \ell \leq \operatorname{dim} \mathcal{K}$, is the least integer such that $\mathcal{R}_{R}^{\ell}$ is a controllability subspace with respect to $\Sigma_{i}$ for all $i \in \mathcal{I}$.

Proof: It follows the same lines of that of Proposition 1.

Definition 4: The subspace $\mathcal{R}_{R}^{*}(\mathcal{K})$, constructed as in Proposition 5 , is called the maximal robust controllability subspace contained in $\mathcal{K}$.

Remark 8: If $\mathcal{V} \subseteq \mathcal{X}$ is a robust controlled invariant subspace for $\Sigma_{\sigma(t)}, \mathcal{R}_{R}^{*}(\mathcal{V})$ is such that the internal dynamics of $\mathcal{V}$ assignable by a friend of $\mathcal{V}$ coincides with the internal dynamics of $\mathcal{R}_{R}^{*}(\mathcal{V})$ : this latter is completely assignable by the same friend, which, as can be shown, is also a friend of $\mathcal{R}_{R}^{*}(\mathcal{V})$.

\section{ACKNOWLEDGMENT}

The authors gratefully acknowledge the anonymous reviewers for the many constructive comments.

\section{REFERENCES}

[1] M. S. Mahmoud, "Generalized $\mathcal{H}_{2}$ control of switched discrete-time systems with unknown delays," Applied Mathematics and Computation, vol. 211, no. 1, pp. 33-44, 2009.

[2] G. S. Deaecto, J. C. Geromel, and J. Daafouz, "Dynamic output feedback $\mathcal{H}_{\infty}$ control of switched linear systems," Automatica, vol. 47, no. 8, pp. 1713-1720, 2011.

[3] M. Balandat, W. Zhang, and A. Abate, "On infinite horizon switched LQR problems with state and control constraints," Systems \& Control Letters, vol. 61, no. 4, pp. 464-471, 2012.

[4] E. Zattoni, A. M. Perdon, and G. Conte, "The output regulation problem with stability for linear switching systems: A geometric approach," Automatica, vol. 49, no. 10, pp. 2953-2962, 2013.

[5] G. Marro, D. Prattichizzo, and E. Zattoni, "A unified setting for decoupling with preview and fixed-lag smoothing in the geometric context," IEEE Transactions on Automatic Control, vol. 51, no. 5, pp. 809-813, 2006.

[6] E. Zattoni, "Decoupling of measurable signals via self-bounded controlled invariant subspaces: Minimal unassignable dynamics of feedforward units for prestabilized systems," IEEE Transactions on Automatic Control, vol. 52, no. 1, pp. 140-143, 2007.
[7] N. Otsuka, "Disturbance decoupling with quadratic stability for switched linear systems," Systems \& Control Letters, vol. 59, no. 6, pp. 349-352, 2010.

[8] G. Conte and A. M. Perdon, "A necessary condition for disturbance decoupling with quadratic stability in switched linear systems," in 19th Mediterranean Conference on Control and Automation, Corfu, Greece, June 20-23, 2011, pp. 1349-1354.

[9] E. Yurtseven, W. P. M. H. Heemels, and M. K. Camlibel, "Disturbance decoupling of switched linear systems," Systems \& Control Letters, vol. 61, no. 1, pp. 69-78, 2012.

[10] E. Zattoni and G. Marro, "A constructive condition for inaccessible signal rejection with quadratic stability in discrete-time linear switching systems," in 52nd IEEE Conference on Decision and Control, Firenze, Italy, December 10-13, 2013, pp. 4650-4655.

[11] H. Lin and P. J. Antsaklis, "Stability and stabilizability of switched linear systems: A survey of recent results," IEEE Transactions on Automatic Control, vol. 54, no. 2, pp. 308-322, 2009.

[12] F. Blanchini and S. Miani, Set-Theoretic Methods in Control. Boston: Birkhäuser, 2008

[13] J.-S. Kim, T.-W. Yoon, and C. De Persis, "Discrete-time supervisory control of input-constrained neutrally stable linear systems via statedependent dwell-time switching," Systems and Control Letters, vol. 56, no. 7-8, pp. 484-492, 2007.

[14] M. Cao and A. S. Morse, "Dwell-time switching," Systems and Control Letters, vol. 59, no. 1, pp. 57-65, 2010.

[15] X. Zhao, L. Zhang, P. Shi, and M. Liu, "Stability of switched positive linear systems with average dwell time switching," Automatica, vol. 48, no. 6, pp. 1132-1137, 2012.

[16] W. Xiang and J. Xiao, "Stabilization of switched continuous-time systems with all modes unstable via dwell time switching," Automatica, vol. 50, no. 3, pp. 940-945, 2014.

[17] A. S. Morse, "Supervisory control of families of linear set-point controllers-Part I: Exact matching," IEEE Transactions on Automatic Control, vol. 41, no. 10, pp. 1413-1431, 1996.

[18] W. M. Wonham, Linear Multivariable Control: A Geometric Approach, 3rd ed. New York: Springer-Verlag, 1985.

[19] G. Basile and G. Marro, Controlled and Conditioned Invariants in Linear System Theory. Englewood Cliffs, New Jersey: Prentice Hall, 1992. [Online]. Available: http://www3.deis.unibo.it/Staff/FullProf/ GiovanniMarro/ctrbm/BOOK12.pdf

[20] A. Banaszuk, M. Kociẹcki, and K. Przyłuski, "The disturbance decoupling problem for implicit linear discrete-time systems," SIAM Journal on Control and Optimization, vol. 28, no. 6, pp. 1270-1293, 1990.

[21] D. Chu and V. Mehrmann, "Disturbance decoupling for descriptor systems by state feedback," SIAM Journal on Control and Optimization, vol. 38, no. 6, pp. 1830-1858, 2000.

[22] G. Conte and A. M. Perdon, "The disturbance decoupling problem for systems over a ring," SIAM Journal on Control and Optimization, vol. 33, no. 3, pp. 750-764, 1995.

[23] C. H. Moog, R. Castro-Linares, M. Velasco-Villa, and L. A. MarquezMartinez, "The disturbance decoupling problem for time-delay nonlinear systems," IEEE Transactions on Automatic Control, vol. 45, no. 2, pp. 305-309, February 2000.

[24] G. Conte, A. M. Perdon, and E. Zattoni, "The disturbance decoupling problem with quadratic stability for LPV systems," in 1st IFAC Workshop on Linear Parameter Varying Systems, Grenoble, France, October 7-9, 2015, pp. 1-6.

[25] E. Zattoni, A. M. Perdon, and G. Conte, "Disturbance decoupling with stability in continuous-time switched linear systems under dwell-time switching," in 19th World Congress of the International Federation of Automatic Control, Cape Town, South Africa, August 24-29, 2014, pp. 164-169.

[26] J. P. Hespanha, "Uniform stability of switched linear systems: Extensions of LaSalle's invariance principle," IEEE Transactions on Automatic Control, vol. 49, no. 4, pp. 470-482, 2004.

[27] G. Basile and G. Marro, "On the robust controlled invariant," Systems \& Control Letters, vol. 9, no. 3, pp. 191-195, 1987.

[28] G. Marro. (2015, October) The Geometric Approach Toolbox. [Online]. Available: http://www3.deis.unibo.it/Staff/FullProf/ GiovanniMarro/geometric.htm\#reftools

[29] G. Chesi, P. Colaneri, J. C. Geromel, R. Middleton, and R. Shorten, "A nonconservative LMI condition for stability of switched systems with guaranteed dwell time," IEEE Transactions on Automatic Control, vol. 57, no. 5, pp. 1297-1302, 2012. 